\title{
Correction to: Numerical investigation of hot ultrasonic assisted turning of aviation alloys
}

\author{
Mehmet Alper Sofuoğlu ${ }^{1}$ Fatih Hayati Çakır ${ }^{2} \cdot$ Selim Gürgen $^{2} \cdot$ Sezan Orak ${ }^{1}$ Melih Cemal Kuşhan ${ }^{1}$
}

Published online: 7 April 2018

(c) The Brazilian Society of Mechanical Sciences and Engineering 2018

\section{Correction to: Journal of the Brazilian Society of Mechanical}

Sciences and Engineering (2018) 40:122

https://doi.org/10.1007/s40430-018-1037-4

The original version of this article unfortunately contained a mistake. Table 1 and Table 2 were incorrect. The correct tables are given here.

Table 1 Johnson Cook coefficients for materials used in simulations $[25,26]$

\begin{tabular}{lllllll}
\hline Materials & $A(\mathrm{MPa})$ & $B(\mathrm{MPa})$ & $C$ & $n$ & $m$ & $\varepsilon_{0}$ \\
\hline Hastelloy-X & 380 & 1200 & 0.012 & 0.55 & 2.5 & 0.001 \\
Ti6Al4V & 724.7 & 683.1 & 0.035 & 0.47 & 1 & 2000 \\
\hline
\end{tabular}

Table 2 Mechanical and thermal properties of materials used in simulations $[29,31,32]$

\begin{tabular}{lll}
\hline Material properties & Ti6Al4V & Hastelloy-X \\
\hline Modulus of elasticity $(\mathrm{MPa})$ & $0.7412 \mathrm{~T}+113,375$ & $209199.4-62.221 \mathrm{~T}$ \\
Thermal expansion $\left(\mathrm{mm} \cdot \mathrm{mm}^{-1}{ }^{\circ} \mathrm{C}^{-1}\right)$ & $3 \times 10^{-9} \mathrm{~T}+7 \times 10^{-6}$ & $1.3 \times 10^{-5}+2.76 \times 10^{-9} \mathrm{~T}$ \\
Thermal conductivity $\left(\mathrm{W} . \mathrm{m}^{-1}{ }^{\circ} \mathrm{C}^{-1}\right)$ & $7.039 \times 10^{(0.0011 \mathrm{~T})}$ & $10.5 \mathrm{e}^{0.001 \mathrm{~T}}$ \\
Emissivity coefficient & 0.7 & 0.85 \\
Poisson's ratio & 0.31 & 0.32 \\
\hline
\end{tabular}

The original article can be found online at https:// doi.org/10.1007/s40430-018-1037-4.

\section{Mehmet Alper Sofuoğlu}

asofuoglu@ogu.edu.tr

1 Department of Mechanical Engineering, Eskişehir Osmangazi University, Eskişehir 26480, Turkey

2 Eskişehir Vocational School, Eskişehir Osmangazi University, Eskişehir 26110, Turkey 